\title{
Is a tight patch necessary after trabeculectomy?
}

\author{
G E Trope, Y M Buys, J Flanagan, L Wang
}

\begin{abstract}
Aims-To determine if posttrabeculectomy patching reduces the prevalence of shallow/flat anterior chambers and pain.

Methods-435 eyes were prospectively randomised to either a double patch with tape or no patch with tape group.

Results-There was no statistically significant difference in flat or shallow anterior chamber rates or pain symptoms between the patch and no patch groups. Conclusion-There is no benefit to patching the post-trabeculectomy eye. (Br F Ophthalmol 1999;83:1006-1007)
\end{abstract}

Shallowing of the anterior chamber due to overfiltration is a well recognised complication of trabeculectomy, ${ }^{1-3}$ with the most common time course being $2-3$ days after surgery. ${ }^{4}$ It is believed by some surgeons that a tight patch reduces the risk of shallow or flat chambers. ${ }^{1}$ The purpose of this study was to determine whether post-trabeculectomy patching influences shallow/flat anterior chamber outcomes and eye discomfort.

\section{Materials and methods}

A total of 435 eyes were prospectively randomised to two treatment groups immediately post-trabeculectomy by the flip of a coin. In all, 202 eyes were left unpatched, while 233 eyes received patching. Patching consisted of taping the lids closed with two strips of tape followed by two flat eye patches or a folded inner patch taped tightly over the eye to apply firm pressure on the eyelids. A plastic shield was then placed over the two patches and taped into place. The

Table 1 Patient demographics

\begin{tabular}{llll}
\hline & $\begin{array}{l}\text { Patch } \\
(n=233)\end{array}$ & $\begin{array}{l}\text { No patch } \\
(n=202)\end{array}$ & Significance \\
\hline Mean age & 63.0 & 62.5 & NS \\
Female & 124 & 92 & NS \\
Male & 109 & 110 & NS \\
RE & 105 & 98 & NS \\
LE & 128 & 104 & NS \\
\hline
\end{tabular}

NS = not significant.

Accepted for publication 26 April 1999

Table 2 Anterior chamber depth versus days after surgery

\begin{tabular}{|c|c|c|c|c|c|c|c|c|}
\hline \multirow[b]{2}{*}{ Postop days } & \multicolumn{4}{|c|}{ Patch $(n=233)$} & \multicolumn{4}{|c|}{ No patch $(n=202)$} \\
\hline & Grade 1 & Grade 2 & Grade 3 & Grade 4 & Grade 1 & Grade 2 & Grade 3 & Grade 4 \\
\hline 1 & $\begin{array}{l}211 \\
(p=0.75)\end{array}$ & $\begin{array}{l}14 \\
(p=0.75)\end{array}$ & $\begin{array}{l}8 \\
(p=1.00)\end{array}$ & $\begin{array}{l}0 \\
(p=1.00)\end{array}$ & 185 & 10 & 7 & 0 \\
\hline 3 & $\begin{array}{l}211 \\
(p=0.75)\end{array}$ & $\begin{array}{l}7 \\
(p=0.10)\end{array}$ & $\begin{array}{l}14 \\
(\mathrm{p}=0.90)\end{array}$ & $\begin{array}{l}1 \\
(p=0.75)\end{array}$ & 178 & 13 & 11 & 0 \\
\hline 7 & $\begin{array}{l}216 \\
(p=0.10)\end{array}$ & $\begin{array}{l}8 \\
(p=0.10)\end{array}$ & $\begin{array}{l}9 \\
(p=0.75)\end{array}$ & $\begin{array}{l}0 \\
(p=1.00)\end{array}$ & 176 & 15 & 11 & 0 \\
\hline 10 & $\begin{array}{l}222 \\
(p=0.90)\end{array}$ & $\begin{array}{l}8^{\star} \\
(p=0.01)\end{array}$ & $\begin{array}{l}3 \\
(p=0.10)\end{array}$ & $\begin{array}{l}0 \\
(p=1.00)\end{array}$ & 194 & 0 & 8 & 0 \\
\hline
\end{tabular}

${ }^{\star}$ Statistical significance. non-patched group had the eyelids taped closed and then covered with a plastic shield. All patches, shields, and tape were removed and patients examined after 24 hours. All surgeries were performed by one of two surgeons from the same institution. ${ }^{5}$ Limbal and fornix based conjunctival flaps were utilised according to surgeon preference. Antimetabolites were used as required. The examiners who graded the anterior chamber depth postoperatively were not masked as to whether the patients were patched or not. The patient's discomfort level in the operated eye was assessed at 24 hours after operation and recorded on a numerical scale which ranged from 1 (least discomfort) to 5 (most discomfort). Anterior chamber depth was assessed on days 1, 3, 7, and 10 respectively. The anterior chamber depth was classified into one of four categories: (1) deep, (2) shallow without iris corneal touch, (3) flat with iris corneal touch, and (4) flat with lens corneal touch.

\section{STATISTICAL ANALYSIS}

The sample size was determined under the assumption that $10 \%$ of patched eyes would have flat chambers, ${ }^{2}$ and the non-patched arm of the study would have double the expected rate of flat chambers (that is, $20 \%$ ). The calculated sample size was 199 eyes per arm of the study, with an $\alpha$ of 0.05 (two tailed) and a power of $80 \%$. Discomfort and flat chamber occurrence rates between the two groups were compared, using $\chi$ analysis $(\mathrm{p}<0.05)$.

\section{Results}

A total of 202 eyes were randomised to non-patching and 233 eyes to patching. Table 1 details the patient demographics. The mean age in the patched group was 63.0 years old versus 62.5 years old in the non-patched group. This was not statistically significant. There was no significant difference between the number of males and females or left and right eyes. Table 2 details the anterior chamber depth versus days after surgery. There was no statistically significant difference between the groups on days $1,3,7$. On day 10 there were significantly more grade 2 chambers in the patch versus the non-patch group $(p=0.01)$. However, there were more grade 3 chambers in the non-patched group on day 10 but this did not reach statistical significance. There was no significant difference in the number of patients who had grade 2 or shallower chambers on day $10(p=0.8)$. There was only one patient who developed lens corneal touch during the study. This occurred on day 3 after trabeculectomy. This patient had been tightly patched. Some $10.7 \%$ of patients in the patched group had significant pain versus $12.8 \%$ in the non- 
patched group (level 4 or 5). This difference was not statistically significant.

\section{Discussion}

This study clearly indicates that it is not necessary to patch an eye tightly after trabeculectomy to prevent discomfort or to prevent a flat or shallow anterior chamber. It is interesting to note that trabeculectomies are relatively painless. In this study only $10.7 \%$ of patients in the patched group and $12.9 \%$ of patients in the non-patched group considered their postoperative discomfort level as status 4 or 5 on day 1. Statistically these data were not significantly different, confirming the fact that tight patching of an eye does not prevent or relieve postoperative pain. It is important to note that during our study the lids in both groups were taped closed after the procedure. We do not know whether this is necessary to relieve pain but in view of the reports with small corneal abrasions, ${ }^{6}$ one could presume that even closing the eyelids may not be necessary after trabeculectomy. Although a prospective study is necessary to confirm this hypothesis, in our practice as we do not use retrobulbar anaesthesia ${ }^{5}$ we no longer tape or patch eyes after trabeculectomy. At the termination of surgery we insert atropine ointment and place a plastic shield over the eye. The eye is left open.

Shallowing of the anterior chamber due to overfiltration is a well recognised complication of trabeculectomy surgery. ${ }^{4}$ As postoperative tight patching is routinely used in many institutions, we thought that deleting the use of a tight patch may increase the risk of shallow or flat chambers. The data from our study, however, do not support this hypothesis. The day 1 data reveal there were $22(10.4 \%)$ patients in the patched group versus $17(9.2 \%)$ in the unpatched group with shallow or flat chambers ( $p>0.05$ ) (grade 2-4). It is well known that chambers often shallow on day 2 or day 3 after trabeculectomy. ${ }^{3}$ There was no statistical difference in the shallow or flat anterior chamber rates at this time between the two groups. Only one $(0.23 \%)$ patient in this study developed lens corneal touch. This was diagnosed on day 3 and resolved by day 7 . This patient was in the patched group but as the flat chamber developed after the patch was removed it is unlikely that the patching played any role in the formation of this complication. It is interesting to note that our flat and shallow anterior chamber rates are less than other reported rates. ${ }^{2-4}$ In one study as many as $50 \%$ of cases had grade 3 (iris cornea touch) shallow anterior chambers. ${ }^{2}$ We think our reduced rate is due to the insertion of three tight scleral sutures into the scleral flap at the end of the procedure. ${ }^{7}$ Our low flat and shallow chamber rates could conceivably have masked a benefit from the use of a patch. Only a very much larger study however would confirm this.

An argument could be made, in spite of the relatively conservative non-parametric analysis used, for the application of a Bonferroni correction for multiple comparisons. Reanalysing our data using this method, would result in an $\alpha$ of 0.003 , thus indicating no significant difference between the patched and nonpatched group at any stage of the study.

In conclusion, this study clearly indicates that patching an eye tightly postoperatively does not reduce the incidence of postoperative pain or flat/shallow chambers. In our practice we no longer patch after trabeculectomy.

We wish to thank the following who helped to collect the data: Drs P Macken, Thillainathan Pathmanathan, C Kranemann, and Elaine Woo.

1 Kao SF, Lichter PR, Musch D. Anterior chamber depth following filtration surgery. Ophthalmic Surg 1989;20:332-6.

2 Steward WC, Bruce Shields M. Management of anterior chamber depth after trabeculectomy. Am $\mathcal{F}$ Ophthalmol 1988;106:41-4

3 Goins K, Smith T, Kinner R, et al. Axial anterior chamber depth after trabeculectomy. Ophthalmologica 1990;200: depth 80 .

4 Suharwardy J, Baranyovit S. The value of peroperative intraocular pressure measurement in trabeculectomy as a predictor of the early postoperative course. Eye 1997;11: predictor

5 Buys Y, Trope GE. Prospective study of sub-Tenon's versus retrobulbar anaesthesia for inpatients and day surgery trabretrobulbar anaesthesia for inpatients and day surg

6 Kaiser PK. A comparison of pressure patching versus no patching for corneal abrasion due to trauma or foreign body removal. Ophthalmology 1994;102:1936-42.

7 Macken P, Buys Y, Trope GE. Laser suture lysis. Br f Ophthalmol 1996;8:398-401. 